\title{
Efficacy and safety comparison of chemotherapies for advanced gastric cancer: A network meta-analysis
}

\author{
Jinping Sun ${ }^{1, *}$, Zheng Ren ${ }^{1, *}$, Xinfang Sun ${ }^{1}$, Hongtao Hou ${ }^{1}$, $\mathrm{Ke} \mathrm{Li}^{1}$, Quanxing Ge ${ }^{1}$ \\ ${ }^{1}$ Department of Digestive Internal Medicine, Huaihe Hospital of Henan University, Kaifeng, 475000, Henan, China \\ *These authors contributed equally to this work
}

Correspondence to: Quanxing Ge, email: whxiadong@126.com

Keywords: advanced gastric cancer, chemotherapies, network meta-analysis, efficacy, safety

Received: February 07, $2017 \quad$ Accepted: April 03, $2017 \quad$ Published: May 11, 2017

Copyright: Jinping Sun et al. This is an open-access article distributed under the terms of the Creative Commons Attribution License 3.0 (CC BY 3.0), which permits unrestricted use, distribution, and reproduction in any medium, provided the original author and source are credited.

\section{ABSTRACT}

Objective: Chemotherapy is one of the commonly used therapies for advanced gastric cancer. In this study, we performed a network meta-analysis on the efficacy and safety of common treatments to give evidences of their relative benefits.

Results: 32 trials with 8550 patients and 20 regimens were included in this study. According to the results of primary outcomes, 5-FU plus OXA, 5-FU plus DOC, CAP plus CIS, CAP plus OXA, S-1 plus OXA and S-1 plus PAC performed well in improving OS and ORR. As for the adverse events, S-1 had a safer effect than other treatments, conversely, 5-FU plus CIS ranked the last. However, there was no regimen with outstanding performances in both efficacy and safety.

Materials and Methods: Studies were searched from database and screened with criteria. The Bayesian framework based network meta-analysis was performed with software $R$ and STATA. Overall survival (OS) and overall response rate (ORR) were considered as primary outcomes while adverse events as secondary outcomes. The outcomes were represented by hazard ratios or odd ratios with $95 \%$ corresponding credible intervals, respectively.

Conclusions: The network meta-analysis suggested that 5-FU plus OXA and 5-FU plus DOC were recommended when efficacy was stressed. S-1 was safest but poorly effective. A regimen, as an excellent combination of efficacy and safety, is still waiting to be discovered.

\section{INTRODUCTION}

Gastric cancer (GC) is a common cancer which leads to a second-high mortality around the world for several decades and caused the estimated 720,000 deaths in 2012 [1]. For the treatment of GC, resection operation has been considered as an optimal option for patients with early stage of GC. However, as the early symptoms of GC are nonspecific, it is hard to diagnose at an early stage and a majority of patients were diagnosed at phase II or III, known as unresectable AGC. So far, gold standard treatment for AGC has not been identified, and patients with AGC have a poor five-year survived rate, making it a challenging task in the clinical research.

Chemotherapy is one of the most common and effective therapies for AGC. A study conducted by Wagner et al. suggested that both overall survival (OS) and the life quality of patients treated with chemotherapy were significantly higher than patients with best supportive care [2]. Among all the chemotherapy drugs, fluorouracil (5-FU) and S-1 were the most conventional ones. 5-FU, which impacts on nucleoside metabolism and leads to cell death, has been used in GC patients since 1960s and its combined regimens have been suggested as effective therapeutic choice since 1980s [3]. 5-FU based combinations, such as 5-FU plus cisplatin (CIS), 5-FU plus adriamycin and mitomycin-C, 5-FU plus irinotecan (IRI), have been reported with an improvement of response rate from $10-15 \%$ (5-FU only) to $40-50 \%$ [4]. S- 1 , an oral combination of tegafur, gimeracil and oteracil, increases the concentration and half-life of 5-FU in plasma, and possibly circumvents its resistance [5]. Theoretically, S-1 based therapy can lead to a higher survival rate and fewer adverse events than 5-FU for patients with AGC. 
However, clinical practice seemed to contradict. For example, meta-analysis in 2016 suggested that S-1 had no difference in terms of OS and progression-free-survival (PFS) compared with 5-FU [6].

In addition to 5-FU and S-1, other drugs were also developed to treat AGC, including platinum drugs, such as CIS and oxaliplatin (OXA), and alcohol drugs, such as paclitaxel (PAC) and docetaxel (DOC), which were usually used in combination with S-1 and 5-FU. A metaanalysis conducted by Liu et al. reported that S-1 based combination therapies, including S-1 plus CIS, S-1 plus IRI and S-1 plus PAC was superior to $\mathrm{S}-1$ were superior to S-1 monotherapy [7].

However, though many new drugs have been used in treating AGC, the progress of chemotherapy is not substantial in the past years mostly due to the drug resistance that tumor cells developed and sever adverse events chemotherapeutic created [8]; besides, the evaluation of existing regimens was insufficient because of the lack of reliable and comprehensive studies, although some efforts have been made. For example, a meta-analysis published in 2014 compared the difference between S-1-based therapy and non-S-1-based therapy and found that S-1-based therapy had no apparent advantage against capecitabine (CAP)-based therapy in efficacy for AGC [9]. Similarly, another meta-analysis in 2016 suggested that S-1 had no difference in terms of OS and progression-free-survival (PFS) compared with 5-FU [6].

Thus, aiming to draw a conclusion and provide reliable evidence, we designed a network meta-analysis with a large amount of data and compared the relative efficacy and safety of common chemotherapies in treating AGC. The relevant drugs include 5-FU, CAP, CIS, DOC, etoposide (ETO), IRI, lentinan (LNT), OXA, and PAC. Note that, because 5-FU is usually used in combination with leucovorin (LV, also named folinic acid) as the basis of most chemotherapy regimens for GC [10], here we regarded 5-FU intensified by leucovorin as an equivalent treatment to 5-FU alone as an adjustment for limited data.

\section{RESULTS}

\section{Baseline characteristics of studies}

At first, 1,929 potentially relevant studies without duplicate were listed after literature search. After being screened with the inclusion criteria, 32 studies with 8,550 patients were finally included without disagreement [10-41]. The included studies, of which 28 were RCTs, 3 retrospective and 1 not specified, were all published between 2004 and 2016. 20 different therapeutic regimens with single or combined use of 10 drugs were contained. The literature searching process was showed in Figure 1. Other basic characteristics of the included studies were presented in Supplementary Table 1.

\section{Primary outcomes}

1-OS

According to the result of network meta-analysis (Figure 2 and Supplementary Table 2), 5-FU plus OXA showed superiority to 13 of the other 19 treatments. 5-FU plus DOC, CAP plus OXA, and CAP+CIS also exhibited desirable performance, significantly better than 10,9 , of the other treatments, respectively. Although not superior to most of the treatments, DOC plus OXA also yielded better results than 6 of the other treatments. .In the meantime, S-1 plus LNT turned out to be the worst choice, followed by 5 -FU and S-1 monotherapy.

\section{2-OS}

The result was similar to that of 1-OS (Figure 2 and Supplementary Table 2). 5-FU plus OXA was superior to most of the treatments while S-1 plus LNT was inferior to most of them followed by 5-FU and S-1. However, the performance of the combination of S-1 and OXA was relatively impressive, exhibiting better performance than 6 other treatments.

\section{3-OS}

The results of 3-OS were presented in Supplementary Table 2 and Figure 3. For the absence of information with respect to 5-FU plus OXA5-FU plus DOC took its place to be the most effective treatment, yielding better results than S-1 plus LNT, 5-FU, 5-FU plus ETO, 5-FU plus IRI and DOC plus CIS. On the other hand, S-1 was significantly worse than S-1 plus OXA and CAP plus CIS.

\section{Overall response rate}

As presented in Supplementary Table 2 and Figure 3, CAP plus CIS and S-1 plus CIS exhibited significantly better performance than 5-FU $(\mathrm{OR}=4.01$, $95 \% \mathrm{CrI}=1.15-14.73 ; \mathrm{OR}=3.35,95 \% \mathrm{CrI}=1.05-10.80$, respectively). However, no significant difference was detected among other treatments.

\section{Adverse events}

With respect to anaemia and anorexia, S-1 performed better than S-1 plus CIS, CAP plus CIS and 5-FU plus CIS. 5-FU was associated with lower risk of diarrhea and fatigue compared to most of other treatments, while IRI plus CIS might lead to more febrile neutropenia and leucopenia events.S-1, S-1 plus IRI and CAP were associated with less nausea events, and S-1 and S-1 plus IRI were also effective in suppressing vomiting events. As for neutropenia, 5-FU, S-1 and IRI plus CIS were associated with lower risk. There was no difference among different treatments with respect to stomatitis and thrombocytopenia. 


\section{Ranking of treatments}

The results of SUCRA were presented in Table 1 , showing the potential ranking probability of these treatments under each outcome. From the ranking of primary outcomes, S-1 plus PAC, CAP plus CIS, 5-FU plus DOC and 5-FU plus OXA were outstanding survival terms and ORR. Nevertheless, as lack of data in 3-OS with respect to 5-FU plus OXA, its efficacy on long-term survival was still uncertain. Additionally, although 5-FU plus DOC presented a high ranking at efficacy, its high risk in both anorexia and diarrhea and insufficient evidence in other adverse events made it seemingly not that reliable.
In the case of adverse events, both S- 1 and CAP exhibited relatively high ranking, which indicated that they were potentially the safest treatments. And with lowest ranking in most safety outcomes, 5-FU plus CIS should be greatly noted. It seemed that there was no ideal treatment which can perform well in both efficacy and safety.

\section{Node-spitting results and net heat plots}

To confirm the consistency between the corresponding results of direct comparison, indirect comparison and network analysis, the node-splitting analysis of outcomes with $P$-value was shown as Supplementary Table 3.
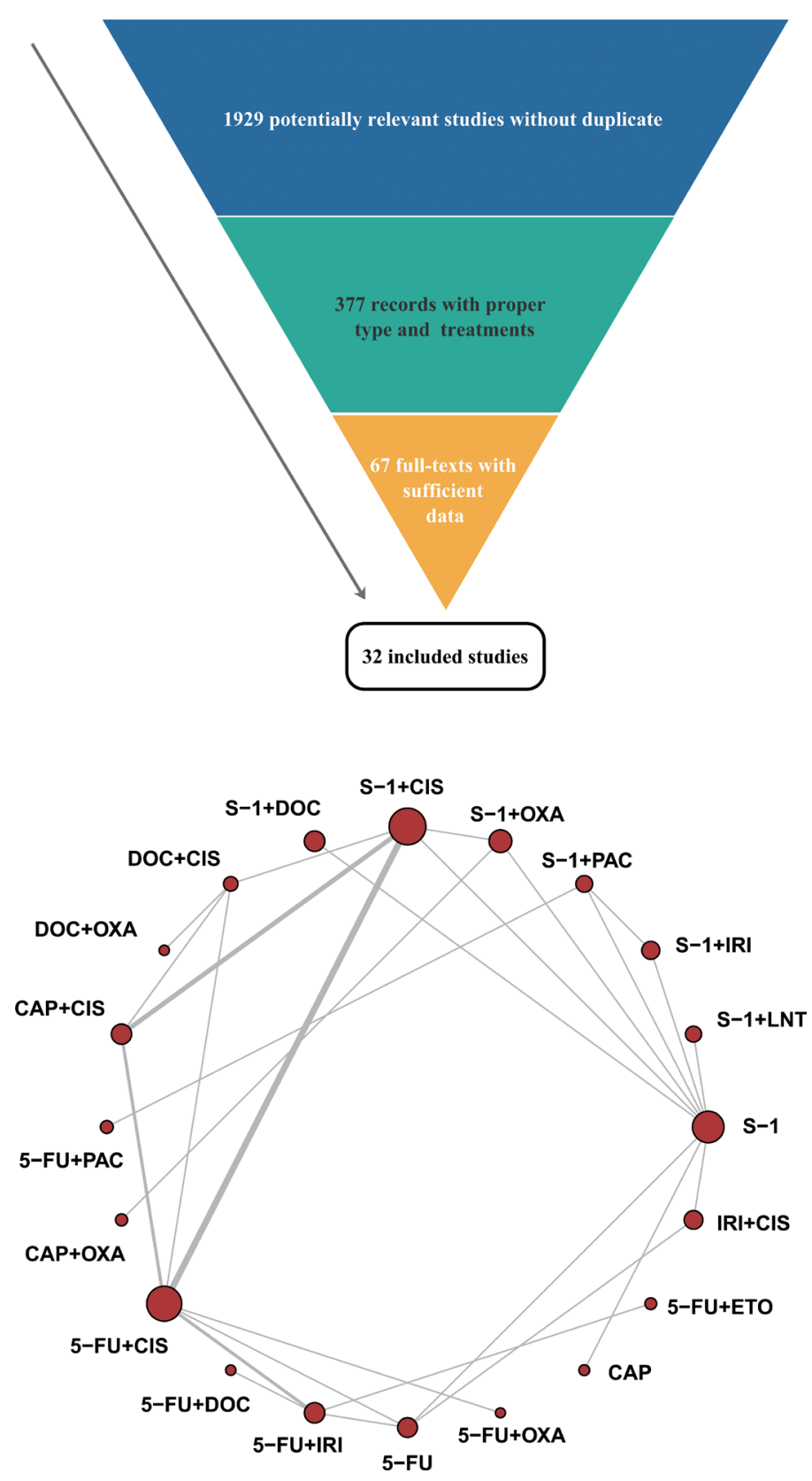

Figure 1: Flow chart and network structure for pain relief. The network plots show direct comparison of different drugs, with node size corresponding to the sample size. 
Table 1: Surface under the cumulative ranking curve (SUCRA) results of OS, ORR and adverse events

\begin{tabular}{|c|c|c|c|c|c|c|c|c|c|c|c|c|c|c|c|}
\hline Drug & $1-\mathrm{OS}$ & 2-OS & 3-os & ORR & Anaemia & Anorexia & Diarrhoea & Fatigue & FN & Leu & Nausea & Neu & Stomatitis & Thr & Vomiting \\
\hline S-1 & 0.134 & 0.197 & 0.320 & 0.329 & 0.795 & 0.870 & 0.435 & 0.712 & 0.770 & 0.676 & 0.850 & 0.802 & 0.649 & 0.696 & 0.767 \\
\hline S-1+LNT & 0.005 & 0.036 & 0.216 & 0.281 & 0.604 & 0.672 & 0.198 & 0.298 & 0.843 & 0.520 & 0.252 & 0.347 & 0.347 & 0.276 & 0.497 \\
\hline S-1+IRI & 0.398 & 0.513 & 0.544 & 0.422 & 0.765 & 0.710 & 0.608 & 0.514 & 0.624 & 0.605 & 0.836 & 0.365 & 0.365 & 0.638 & 0.875 \\
\hline S-1+PAC & 0.743 & 0.737 & 0.698 & 0.591 & 0.342 & 0.536 & 0.612 & 0.551 & 0.825 & 0.707 & 0.440 & 0.502 & 0.502 & 0.767 & 0.385 \\
\hline S-1+OXA & 0.640 & 0.800 & 0.738 & 0.313 & 0.602 & 0.338 & 0.443 & 0.491 & 0.662 & 0.538 & 0.355 & 0.585 & 0.585 & 0.292 & 0.619 \\
\hline $\mathrm{S}-1+\mathrm{CIS}$ & 0.502 & 0.527 & 0.626 & 0.702 & 0.242 & 0.215 & 0.384 & 0.335 & 0.403 & 0.239 & 0.280 & 0.647 & 0.647 & 0.273 & 0.366 \\
\hline S-1+DOC & 0.298 & 0.421 & 0.505 & 0.746 & - & 0.732 & 0.602 & 0.592 & 0.276 & 0.245 & 0.684 & 0.386 & 0.386 & - & 0.639 \\
\hline $\mathrm{DOC}+\mathrm{CIS}$ & 0.536 & 0.399 & 0.099 & 0.516 & - & - & - & - & - & - & - & - & - & - & - \\
\hline DOC + OXA & 0.825 & 0.567 & 0.187 & 0.588 & - & - & - & - & - & - & - & - & - & - & - \\
\hline CAP+CIS & 0.784 & 0.719 & 0.794 & 0.780 & 0.188 & 0.318 & 0.616 & 0.055 & 0.568 & 0.348 & 0.303 & 0.611 & 0.611 & 0.352 & 0.323 \\
\hline 5-FU+PAC & 0.571 & 0.634 & - & 0.476 & 0.123 & 0.633 & 0.606 & 0.682 & - & 0.824 & 0.360 & 0.345 & 0.345 & 0.887 & 0.201 \\
\hline CAP+OXA & 0.865 & 0.837 & 0.763 & 0.413 & 0.916 & 0.406 & 0.453 & - & - & 0.704 & 0.242 & - & - & 0.335 & 0.366 \\
\hline 5-FU+CIS & 0.419 & 0.397 & 0.538 & 0.461 & 0.293 & 0.272 & 0.599 & 0.280 & 0.219 & 0.217 & 0.139 & 0.247 & 0.247 & 0.270 & 0.223 \\
\hline 5-FU+DOC & 0.869 & 0.863 & 0.904 & 0.604 & - & 0.076 & 0.116 & - & - & - & - & - & - & - & - \\
\hline 5-FU+IRI & 0.561 & 0.467 & 0.623 & 0.697 & 0.521 & 0.453 & 0.185 & - & 0.397 & 0.350 & 0.423 & 0.513 & 0.513 & 0.658 & 0.371 \\
\hline $5-\mathrm{FU}$ & 0.062 & 0.055 & 0.185 & 0.160 & 0.549 & 0.843 & 0.944 & 0.935 & 0.500 & 0.831 & 0.776 & 0.353 & 0.353 & 0.379 & - \\
\hline 5-FU+OXA & 0.934 & 0.934 & - & 0.764 & - & 0.518 & 0.726 & 0.689 & - & - & - & - & - & - & - \\
\hline CAP & 0.284 & 0.405 & - & 0.325 & 0.816 & 0.867 & 0.272 & - & 0.563 & 0.627 & 0.951 & 0.599 & 0.599 & 0.634 & 0.885 \\
\hline 5-FU+ETO & 0.170 & 0.198 & 0.409 & 0.330 & 0.244 & 0.127 & 0.840 & - & 0.281 & - & 0.701 & - & - & 0.545 & 0.483 \\
\hline IRI+CIS & 0.401 & 0.296 & 0.351 & - & - & 0.416 & 0.361 & 0.366 & 0.069 & 0.070 & 0.407 & 0.852 & 0.852 & - & - \\
\hline
\end{tabular}

Note: $\mathrm{FN}=$ Febrile Neutropenia; Leu = Leucopenia; Neu = Neutropenia; Thr= Thrombocytopenia. The red color indicates good ranking, while green means bad result.

A $P$-value $<0.05$ indicates a significant inconsistency. According to the results, most of the network analyses were consistent with the direct comparison with a $P$-value lager than 0.05 . However, there were several exceptions: when compared with S-1 plus OXA in ORR, the nodesplitting results of S-1 and S-1 plus CIS showed significant inconsistency ( $P$-value $=0.024$ and 0.023 , respectively). Besides, the comparison of S-1 versus S-1 plus IRI in terms of leucopenia was also indicated as significant inconsistency with a $P$-value of 0.034 . In addition, the net heat plots of primary outcomes were showed as Figure 4. Similar with the result of node-spitting, the net heat plots illustrate little heterogeneity of the evidence.

\section{DISCUSSION}

AGC keeps a high incidence and mortality around the world. Although many kinds of chemotherapies have been utilized for treating it, the OS is still poor because of the high probability of lurks and recurrence. To give valuable suggestions for treatments through comparing their efficacy and safety, we conducted the network metaanalysis among 20 chemotherapy regimens with 10 drugs commonly used for AGC, including S-1 based therapies, 5-FU based therapies and others.

The results demonstrated that 5-FU plus OXA may have a best efficacy in treating patients with AGC. OXA is a 3 rd generation platinum compound with a better accumulation and safety profile in tumor cells than CIS. The mechanism of its antitumor action is suggested as its interference on DNA adducts formation by affecting DNA damage repairing proteins, transcription factors and DNA polymerases [42]. It has been verified that the combination chemotherapy of OXA and 5-FU is effective in 5-FU- and CIS-resistant cell lines, meanwhile 5-FU can increase the expression of $M R P 2 / A B C C 2$ pathway and induce a hypersensitivity to OXA [42, 43]. Results of this NMA supported its efficacy in improving OS and ORR; furthermore, it had a good performance in known adverse events including diarrhea and fatigue, which suggested that the combination regimen of 5-FU and OXA may have a great potential as a mainstream chemotherapy for AGC. However, due to the lack of evidence, most outcomes concerning safety under this treatment were not included in this study. Therefore, it should be further evaluated before any clinical commendation was given. 
Similar to 5-FU plus OXA, the combination of 5-FU and DOC was outstanding for efficacy in both OS and ORR, but its risk in inducing anorexia and diarrhea was higher than most of the other treatments. DOC is one of the new chemotherapeutic drugs widely used for tumors since 1990 s, and its promising antitumor activity in several cancers has been verified by previous studies, both as mono therapy and combined with other drugs [44]. It is also believed that the combination regimen DCF (DOC/ CIS/5-FU) could become a standard therapy for advanced, recurrent and metastatic GC [44]. Nevertheless, along with its good efficacy, the high risk of toxicity, especially gastrointestinal toxicity, of double and triple DOC based chemotherapies were also indicated by Tetzlaff et al. [45]. According to our network meta-analysis, the incidence of anorexia and diarrhea among patients treated with 5-FU plus DOC was significantly higher than that of 5-FU mono therapy, which was in line with previous studies. Therefore, dosage must be administrated to control the adverse events in clinical use. To evaluate the efficacy and safety of 5-FU plus DOC for AGC accurately, more experimental data are needed.

Focusing on the adverse events, this NMA indicated that mono therapy of S-1 exhibited the best performance in preventing adverse event, while its efficacy was nearly the worst. On the other hand, S-1 based polytherapies, including S-1 plus PAC, S-1 plus OXA and S-1 plus CIS, exhibited better performance in efficacy, with an increasing risk of adverse events. In fact, there have already been several previous studies focusing on the

\section{1 year-OS}
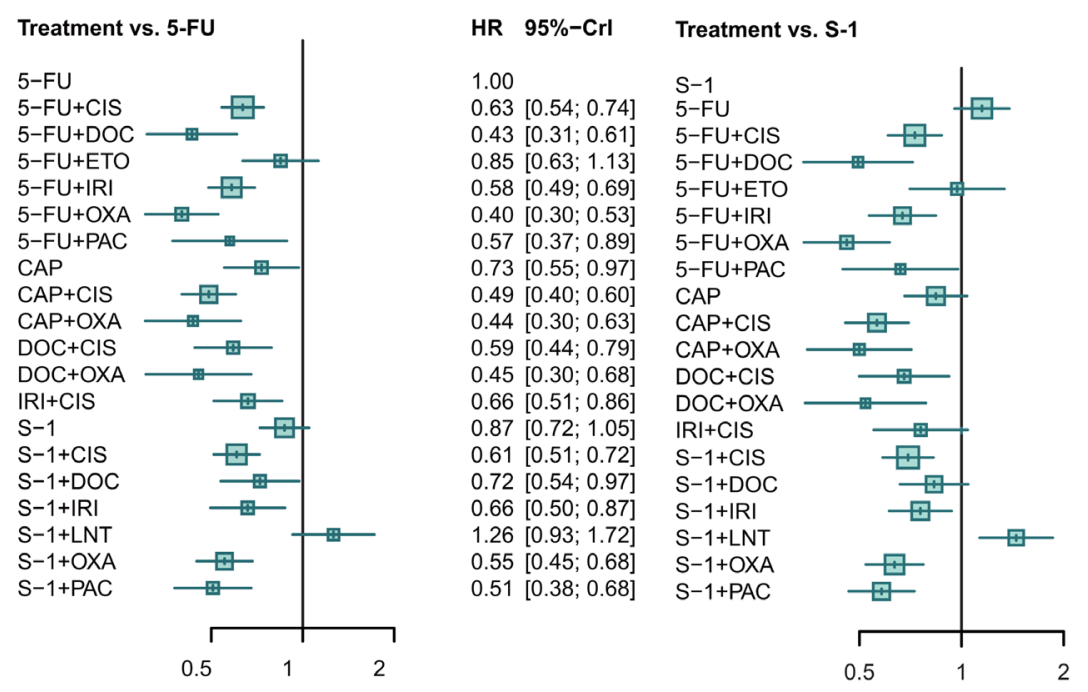

\section{2 year-OS}

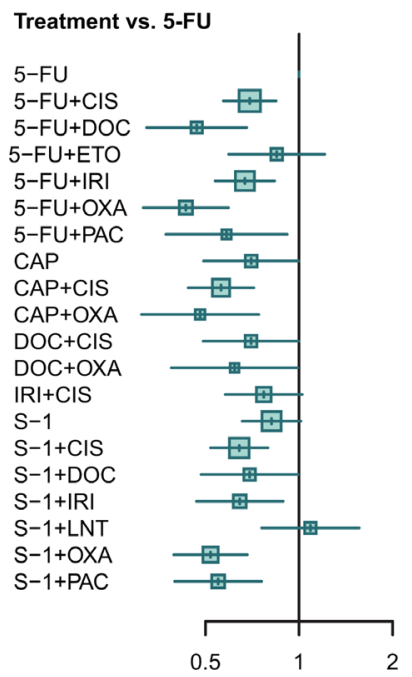

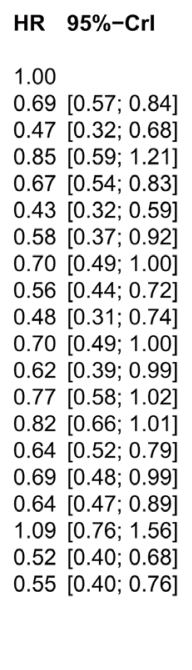

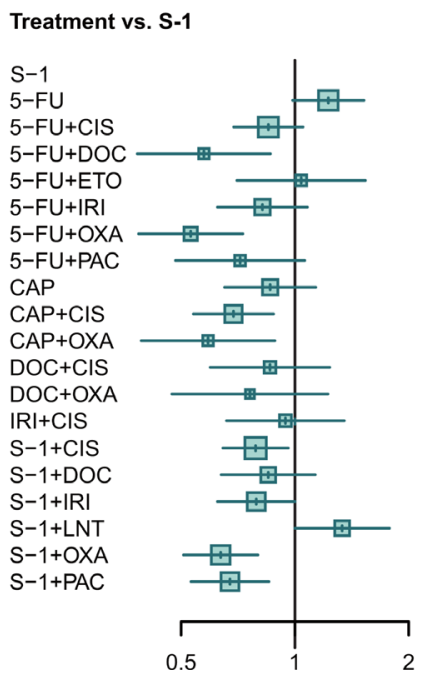

Figure 2: Forest plots for 1 year-OS and 2 year -OS. Hazard ratios (HRs) with 95\% credible interval (CrIs) indicate the relative efficacy.

HR $95 \%-C r I$

1.00

$1.15[0.95 ; 1.38]$

$0.73[0.61 ; 0.87]$

$0.50[0.34 ; 0.72]$

$0.97[0.70 ; 1.34]$

$0.67[0.53 ; 0.84]$

$0.46[0.34 ; 0.61]$

$0.66[0.45 ; 0.98]$

$0.84[0.68 ; 1.04]$

$0.56[0.45 ; 0.70]$

$0.50[0.35 ; 0.71]$

0.68 [0.50;0.92]

$0.52[0.35 ; 0.78]$

$0.76[0.55 ; 1.04]$

$0.70[0.59 ; 0.83]$

$0.83[0.66 ; 1.05]$

$0.76[0.61 ; 0.94]$

$1.45[1.13 ; 1.86]$

.63 [0.52; 0.77$]$

$0.58[0.46 ; 0.73]$

$1.23[0.99 ; 1.52]$

$0.85[0.69 ; 1.05]$

0.57 [0.38; 0.86]

$1.04[0.70 ; 1.54]$

$0.82[0.62 ; 1.08]$

$0.53[0.39 ; 0.73]$

$0.72[0.48 ; 1.06]$

$0.86[0.65 ; 1.14]$

$0.69[0.54 ; 0.88]$

0.59 [0.39; 0.89$]$

$0.86[0.60 ; 1.24]$

$0.76[0.47 ; 1.22]$

$0.94[0.66 ; 1.35]$

$0.79[0.64 ; 0.96]$

$0.85[0.64 ; 1.13]$

$0.79[0.62 ; 1.00]$

$1.33[1.00 ; 1.78]$

$0.64[0.51 ; 0.80]$

0.67 [0.53;0.85] 
difference between mono therapy and combination therapy of S-1: a meta-analysis conducted by Liu et al. analyzed four RCTs and indicated that S-1 based combination therapies led to a better OS, PFS and ORR than the mono therapy S-1 in treating AGC, but the mono therapy had a lower incidence of leucopenia, neutropenia and diarrhea, which was consistent with our results [46]. In fact, before this study, several previous network meta-analyses of chemotherapies for AGC have been published, however, most of them focused on the comparison of general types of regimens, such as S-1 based and 5-FU based, but not on certain treatments. For instance, a study published in 2016 suggested that both S-1 based and capecitabine based regimens had a significant improvement of OS when compared with 5-FU based regimens [47]. In this study, the data of specific therapies were analyzed to screen out the optimal regimens with relatively better efficacy and safety, which was a further attempt. The combination of direct and indirect comparison with a relatively large sample size and consistency added support for the reliability to our results.

However, several limitations of our analysis should also be taken into account. The node-splitting results and heat plots showed consistency between direct and indirect analysis of most of comparisons except that of S-1 plus OXA versus S-1 in ORR, S-1 plus OXA versus

\section{3 year-OS}

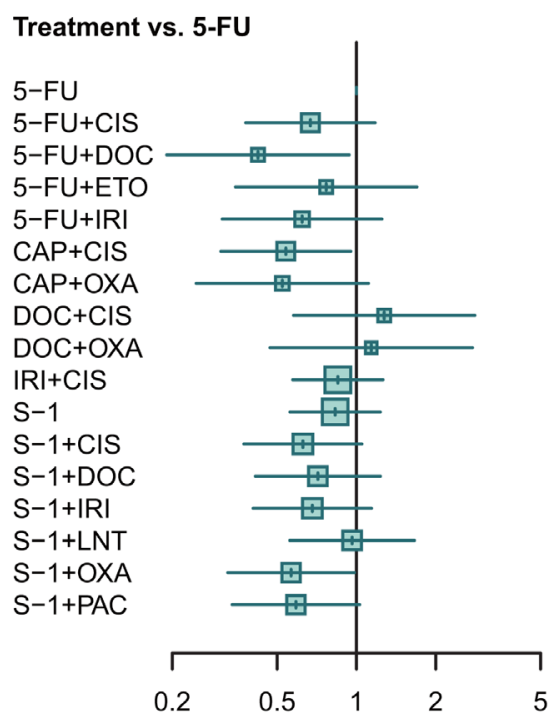

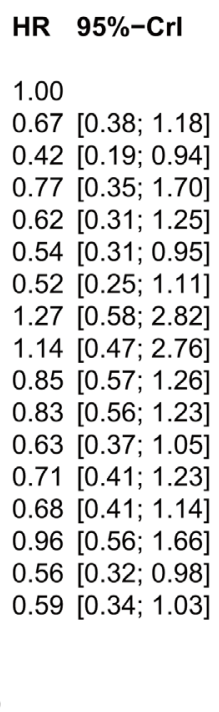

HR $95 \%-\mathrm{Crl}$

Treatment vs. S-1

$\mathrm{S}-1$
$5-\mathrm{FU}$

$5-\mathrm{FU}+\mathrm{CIS}$

5-FU+DOC

5-FU+ETO

5-FU+IRI

$\mathrm{CAP}+\mathrm{CIS}$

$\mathrm{CAP}+\mathrm{OXA}$

$\mathrm{DOC}+\mathrm{CIS}$

$\mathrm{DOC}+\mathrm{OXA}$

IRI+CIS

$\mathrm{S}-1+\mathrm{CIS}$

S-1+DOC

$S-1+|R|$

S-1+LNT

S-1+OXA

$\mathrm{S}-1+\mathrm{PAC}$

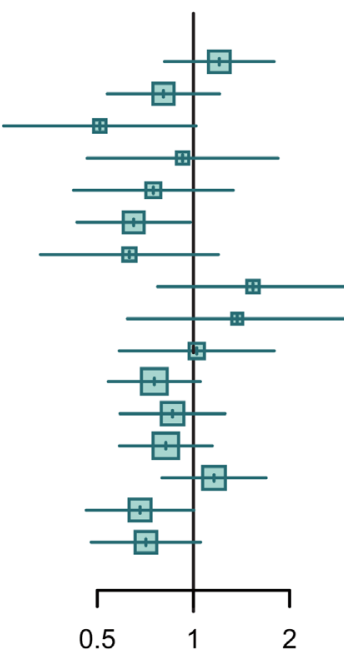

HR $\quad 95 \%-C r l$

1.00

$1.20 \quad[0.81 ; 1.79]$

$0.81 \quad[0.54 ; 1.21]$

$0.51 \quad[0.25 ; 1.02]$

$0.92 \quad[0.46 ; 1.84]$

$0.75 \quad[0.42 ; 1.33]$

$0.65 \quad[0.43 ; 0.98]$

$0.63 \quad[0.33 ; 1.20]$

$1.54 \quad[0.77 ; 3.05]$

$1.37 \quad[0.62 ; 3.03]$

$1.02 \quad[0.59 ; 1.79]$

$0.75 \quad[0.54 ; 1.05]$

$0.86 \quad[0.59 ; 1.26]$

$0.82 \quad[0.59 ; 1.14]$

$1.16 \quad[0.80 ; 1.69]$

$0.68 \quad[0.46 ; 1.00]$

$0.71 \quad[0.48 ; 1.05]$

\section{ORR}

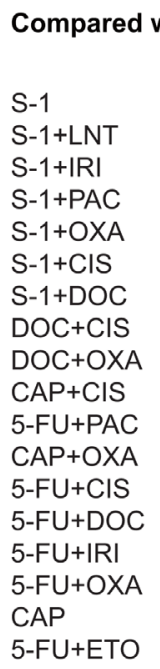

th $5-F U$

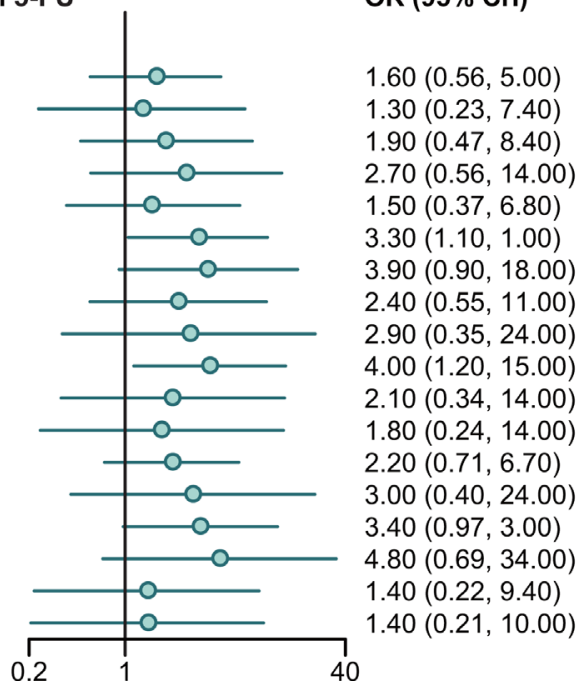

Compared with S-1

S-1+LNT

$\mathrm{S}-1+\mid \mathrm{RI}$

S-1+PAC

S-1+OXA

$\mathrm{S}-1+\mathrm{CIS}$

S-1+DOC

$\mathrm{DOC}+\mathrm{CIS}$

$\mathrm{DOC}+\mathrm{OXA}$

$\mathrm{CAP}+\mathrm{CIS}$

5-FU+PAC

$\mathrm{CAP}+\mathrm{OXA}$

5-FU+CIS

$5-F U+D O C$

5-FU+IRI

5-FU

5-FU+OXA

CAP

5-FU+ETO

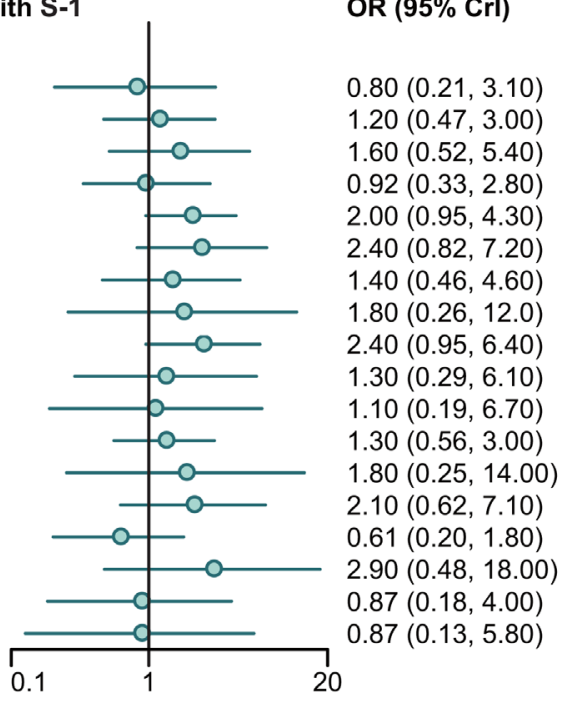

Figure 3: Forest plots for 3 year-OS and ORR. Hazard ratios (HRs) and odds ratios (ORs) with 95\% credible interval (CrIs) indicate the relative efficacy. 
S-1 plus CIS in ORR, and S-1 versus S-1 plus IRI in leucopenia. A main source of the inconsistency was likely to be insufficiency of direct evidence. What's more, characteristics of included trials like sample size can make the indirect results quite different from the direct results in a way. To reduce the inconsistency and improve the reliability of the results, more pairwise comparison should be included in the further studies. Besides, results could be influenced by trial design as well. Several of our included trials were not randomized. And in some included trials, leucovorin was taken with 5-FU as an enhancer, the benefit solely brought by leucovorin was not separated or evaluated in this study. With a larger number of individual studies, the further analysis may get a more reliable result by narrowing the type of trials and classifying treatments more meticulously. Moreover, as the course of disease and dosage varied from patient to patient, the corresponding adjustment may contribute to a more accurate analysis.

In conclusion, our study demonstrated that 5-FU plus OXA and 5-FU plus DOC were the best two regimens for
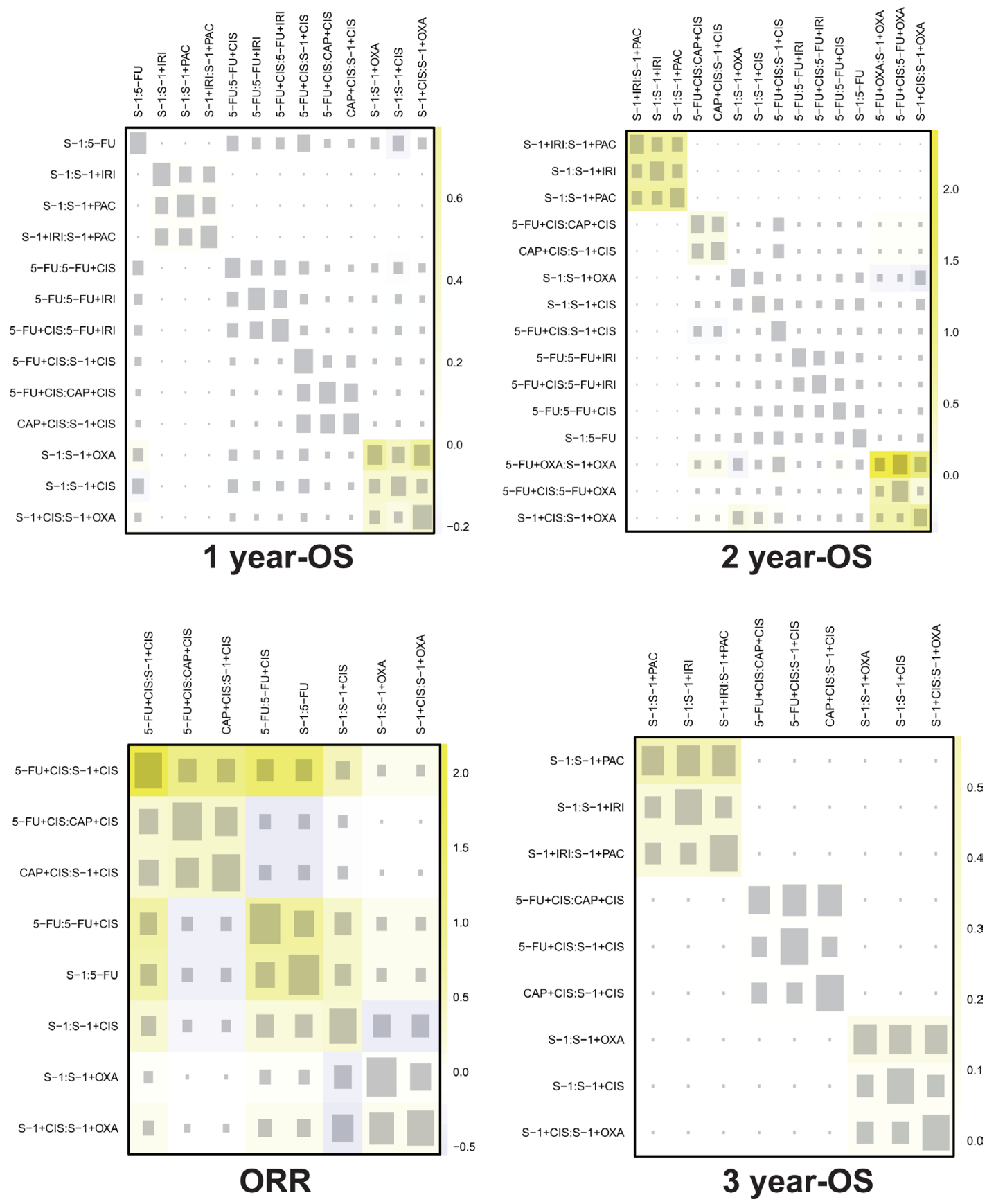

Figure 4: Heat plots for OS and ORR. The size of the gray squares indicates the contribution of the direct evidence (shown in the column) to the network evidence (shown in the row). The colors are associated with the change in inconsistency between direct and indirect evidence (shown in the row). Blue colors indicate an increase of inconsistency and warm colors indicate a decrease. 
AGC when only considering efficacy, and S-1 was proved to be the safest one. However, the risk of 5-FU plus OXA or 5-FU plus DOC to cause adverse events still remained unclear, and S-1 had a relatively lower efficacy for treatment. A regimen, which has an excellent combination of efficacy and safety, still remains to be discovered.

\section{MATERIALS AND METHODS}

\section{Literature search}

We conducted a comprehensive search on databases including China National Knowledge Internet (CNKI), Embase and Pubmed for literature published between January 1st, 2000 and November 1st, 2016. Literature were identified with key words including "stomach neoplasms", "chemotherapy", "capecitabine", "cisplatin", "docetaxel", "fluorouracil", "irinotecan", "lentinan", "oxaliplatin", "paclitaxel", "etoposide", "S-1" and their synonyms. Duplicate and irrelevant studies were excluded manually after being verified.

\section{Inclusion}

To ensure the reliability of our outcomes, two investigators independently screened the title and abstract of the retrieved studies. Only studies fulfilling the following inclusion criteria were included: 1) diagnosis of AGC among all patients of the study should be confirmed; 2) data of patients, treatments and outcomes should be sufficient; 3 ) the study should be conducted with one or more pairwise comparison between the included treatments; 4) the outcomes of study should include at least one of the included primary outcomes or secondary outcomes.

\section{Outcomes}

Primary outcomes included 1, 2, 3-year OS (1-OS, 2-OS, 3-OS) and overall response rate (ORR), which were used to measure the efficacy of different treatments. Secondary outcomes were adverse events (grade $\geq 3$ ) including anemia, anorexia, diarrhea, fatigue, febrile neutropenia, leucopenia, nausea, neutropenia, stomatitis, thrombocytopenia and vomiting, which were measure the safety of different treatments.

\section{Data extraction}

The data of included studies were extracted by two independent investigators. The baseline characteristics of all studies, including author, published year, study design, age and gender of patients, group size and treatments were collected. Besides, the data for outcomes as mentioned above were also extracted and recorded. Any discrepancy between the two investigators would be resolved by carrying a discussion to reach a consensual conclusion.

\section{Data analysis}

Network meta-analysis was performed with Bayesian framework in software R (V3.3.1) and STATA (V13.0) based on our design. Primary outcomes including 1-OS, 2-OS and 3-OS were represented by hazard ratios (HRs) with 95\% corresponding credible intervals (CrIs), while ORR and adverse events were represented by odds ratios (ORs) with 95\% corresponding CrIs. Moreover, surface under the cumulative ranking curve (SUCRA) was calculated to show the potential ranking probability of each treatment under each outcome and to identify proper recommended treatments. In addition, node-splitting plots and net heat plots were computed to analyze the inconsistency between direct and indirect evidence.

\section{ACKNOWLEDGMENTS}

None

\section{CONFLICTS OF INTEREST}

The authors have no relevant conflicts of interest to disclose.

\section{FUNDING}

None

\section{REFERENCES}

1. Van Cutsem E, Sagaert X, Topal B, Haustermans K, Prenen H. Gastric cancer. Lancet. 2016; 388:2654-2664.

2. Wagner AD, Grothe W, Haerting J, Kleber G, Grothey A, Fleig WE. Chemotherapy in advanced gastric cancer: a systematic review and meta-analysis based on aggregate data. J Clin Oncol. 2006; 24:2903-2909.

3. Zhang $\mathrm{N}$, Yin $\mathrm{Y}, \mathrm{Xu}$ SJ, Chen WS. 5-Fluorouracil: mechanisms of resistance and reversal strategies. Molecules. 2008; 13:1551-1569.

4. Douillard JY, Cunningham D, Roth AD, Navarro M, James RD, Karasek P, Jandik P, Iveson T, Carmichael J, Alakl M, Gruia G, Awad L, Rougier P. Irinotecan combined with fluorouracil compared with fluorouracil alone as firstline treatment for metastatic colorectal cancer: a multicentre randomised trial. Lancet. 2000; 355:1041-1047.

5. Krasniqi E, Pellicori S, Formica V. Emerging role of S-1 in gastric cancer. Indian J Med Paediatr Oncol. 2015; 36:219 228.

6. Ter Veer E, Mohammad NH, Lodder P, Ngai LL, Samaan M, van Oijen MG, van Laarhoven HW. The efficacy and safety of S-1-based regimens in the first-line treatment of advanced gastric cancer: a systematic review and metaanalysis. Gastric Cancer. 2016; 19:696-712. 
7. Liu H, Chen X, Sun J, Gao P, Song Y, Zhang N, Lu X, Xu H, Wang Z. The efficacy and toxicity of paclitaxel plus S-1 compared with paclitaxel plus 5-FU for advanced gastric cancer: a PRISMA systematic review and meta-analysis of randomized controlled trials. Medicine (Baltimore). 2014; 93:e164.

8. Sun W, Yan L. Gastric cancer: current and evolving treatment landscape. Chin J Cancer. 2016; 35:83.

9. Yang J, Zhou Y, Min K, Yao Q, Xu CN. S-1-based vs non-S1-based chemotherapy in advanced gastric cancer: a metaanalysis. World J Gastroenterol. 2014; 20:11886-11893.

10. Moehler M, Eimermacher A, Siebler J, Hohler T, Wein A, Menges M, Flieger D, Junginger T, Geer T, Gracien E, Galle PR, Heike M. Randomised phase II evaluation of irinotecan plus high-dose 5-fluorouracil and leucovorin (ILF) vs 5-fluorouracil, leucovorin, and etoposide (ELF) in untreated metastatic gastric cancer. Br J Cancer. 2005; 92:2122-2128.

11. Ajani JA, Buyse M, Lichinitser M, Gorbunova V, Bodoky G, Douillard JY, Cascinu S, Heinemann V, Zaucha R, Carrato A, Ferry D, Moiseyenko V. Combination of cisplatin/S-1 in the treatment of patients with advanced gastric or gastroesophageal adenocarcinoma: Results of noninferiority and safety analyses compared with cisplatin/5-fluorouracil in the First-Line Advanced Gastric Cancer Study. Eur J Cancer. 2013; 49:3616-3624.

12. Ajani JA, Rodriguez W, Bodoky G, Moiseyenko V, Lichinitser M, Gorbunova V, Vynnychenko I, Garin A, Lang I, Falcon S. Multicenter phase III comparison of cisplatin/S-1 with cisplatin/infusional fluorouracil in advanced gastric or gastroesophageal adenocarcinoma study: the FLAGS trial. J Clin Oncol. 2010; 28:1547-1553.

13. Boku N, Yamamoto S, Fukuda H, Shirao K, Doi T, Sawaki A, Koizumi W, Saito H, Yamaguchi K, Takiuchi H, Nasu J, Ohtsu A. Fluorouracil versus combination of irinotecan plus cisplatin versus S-1 in metastatic gastric cancer: a randomised phase 3 study. Lancet Oncol. 2009; 10:1063-1069.

14. Bouche O, Raoul JL, Bonnetain F, Giovannini M, Etienne PL, Lledo G, Arsene D, Paitel JF, GuerinMeyer V, Mitry E, Buecher B, Kaminsky MC, Seitz JF, et al. Randomized multicenter phase II trial of a biweekly regimen of fluorouracil and leucovorin (LV5FU2), LV5FU2 plus cisplatin, or LV5FU2 plus irinotecan in patients with previously untreated metastatic gastric cancer: a Federation Francophone de Cancerologie Digestive Group Study--FFCD 9803. J Clin Oncol. 2004; 22:4319-4328.

15. Dank M, Zaluski J, Barone C, Valvere V, Yalcin S, Peschel C, Wenczl M, Goker E, Cisar L, Wang K, Bugat R. Randomized phase III study comparing irinotecan combined with 5-fluorouracil and folinic acid to cisplatin combined with 5-fluorouracil in chemotherapy naive patients with advanced adenocarcinoma of the stomach or esophagogastric junction. Ann Oncol. 2008; 19:1450-1457.

16. Gubanski M, Johnsson A, Fernebro E, Kadar L, Karlberg I, Flygare P, Berglund A, Glimelius B, Lind PA. Randomized phase II study of sequential docetaxel and irinotecan with 5-fluorouracil/folinic acid (leucovorin) in patients with advanced gastric cancer: the GATAC trial. Gastric Cancer. 2010; 13:155-161.

17. Huang D, Ba Y, Xiong J, Xu N, Yan Z, Zhuang Z, Yu Z, Wan H, Zhang Y, Deng T, Zheng R, Guo Z, Hu C, et al. A multicentre randomised trial comparing weekly paclitaxel + S-1 with weekly paclitaxel +5 -fluorouracil for patients with advanced gastric cancer. Eur J Cancer. 2013; 49:2995-3002.

18. Jeung HC, Rha SY, Im CK, Shin SJ, Ahn JB, Yang WI, Roh JK, Noh SH, Chung HC. A randomized phase 2 study of docetaxel and S-1 versus docetaxel and cisplatin in advanced gastric cancer with an evaluation of SPARC expression for personalized therapy. Cancer. 2011; 117:2050-2057.

19. Kang YK, Kang WK, Shin DB, Chen J, Xiong J, Wang J, Lichinitser M, Guan Z, Khasanov R, Zheng L, PhilcoSalas M, Suarez T, Santamaria J, et al. Capecitabine/ cisplatin versus 5-fluorouracil/cisplatin as first-line therapy in patients with advanced gastric cancer: a randomised phase III noninferiority trial. Ann Oncol. 2009; 20:666-673.

20. Kim GM, Jeung HC, Rha SY, Kim HS, Jung I, Nam BH, Lee KH, Chung HC. A randomized phase II trial of S-1oxaliplatin versus capecitabine-oxaliplatin in advanced gastric cancer. Eur J Cancer. 2012; 48:518-526.

21. Kim YS, Sym SJ, Park SH, Park I, Hong J, Ahn HK, Park J, Cho EK, Lee WK, Chung M, Lee JH, Shin DB. A randomized phase II study of weekly docetaxel/cisplatin versus weekly docetaxel/oxaliplatin as first-line therapy for patients with advanced gastric cancer. Cancer Chemother Pharmacol. 2014; 73:163-169.

22. Kobayashi M, Tsuburaya A, Nishikawa K, Kawada J, Namikawa T, Fukushima R, Kojima H, Tanabe K, Yamaguchi K, Yoshino S, Takahashi M, Hirabayashi N, Sato S, et al. A randomized phase II trial of capecitabine plus cisplatin (XP) versus S-1 plus cisplatin (SP) as a first-line treatment for advanced gastric cancer: XP ascertainment versus SP randomized PII trial (XParTS II). Journal of Clinical Oncology. 2015; 33.

23. Koizumi W, Kim YH, Fujii M, Kim HK, Imamura H, Lee KH, Hara T, Chung HC, Satoh T, Cho JY, Hosaka H, Tsuji A, Takagane A, et al. Addition of docetaxel to S-1 without platinum prolongs survival of patients with advanced gastric cancer: a randomized study (START). J Cancer Res Clin Oncol. 2014; 140:319-328.

24. Koizumi W, Narahara H, Hara T, Takagane A, Akiya T, Takagi M, Miyashita K, Nishizaki T, Kobayashi O, Takiyama W, Toh Y, Nagaie T, Takagi S, et al. S-1 plus cisplatin versus S-1 alone for first-line treatment of advanced gastric cancer (SPIRITS trial): a phase III trial. Lancet Oncol. 2008; 9:215-221.

25. Komatsu Y, Takahashi Y, Kimura Y, Oda H, Tajima Y, Tamura S, Sakurai J, Wakasugi T, Tatebe S, Takahashi M, Sakata Y, Kitajima M, Sakamoto J, et al. Randomized phase II trial of first-line treatment with tailored irinotecan and S-1 therapy versus S-1 monotherapy for advanced or recurrent 
gastric carcinoma (JFMC31-0301). Anticancer Drugs. 2011; 22:576-583.

26. Lee JL, Kang YK, Kang HJ, Lee KH, Zang DY, Ryoo BY, Kim JG, Park SR, Kang WK, Shin DB, Ryu MH, Chang HM, Kim TW, et al. A randomised multicentre phase II trial of capecitabine vs S-1 as first-line treatment in elderly patients with metastatic or recurrent unresectable gastric cancer. Br J Cancer. 2008; 99:584-590.

27. Li YH, Qiu MZ, Xu JM, Sun GP, Lu HS, Liu YP, Zhong MZ, Zhang HL, Yu SY, Li W, Hu XH, Wang JJ, Cheng Y, et al. S-1 plus cisplatin versus fluorouracil plus cisplatin in advanced gastric or gastro-esophageal junction adenocarcinoma patients: a pilot study. Oncotarget. 2015; 6:35107-35115. doi: 10.18632/oncotarget.5959.

28. Lim DH, Park SH, Park KW, Kang JH, Oh SY, Hwang IG, Kwon JM, Lee SC, Lee HY, Kim HS, Lim HY, Kang WK. Retrospective analyses of cisplatin-based doublet combination chemotherapy in patients with advanced gastric cancer. BMC Cancer. 2010; 10:583.

29. Lu Y, Liu Z, Zhang J. S-1 plus oxaliplatin vs. S-1 as first-line treatment in patients with previously untreated advanced gastric cancer: a randomized phase II study. J Chemother. 2014; 26:159-164.

30. Narahara H, Iishi H, Imamura H, Tsuburaya A, Chin K, Imamoto H, Esaki T, Furukawa H, Hamada C, Sakata Y. Randomized phase III study comparing the efficacy and safety of irinotecan plus S-1 with S-1 alone as first-line treatment for advanced gastric cancer (study GC0301/TOP002). Gastric Cancer. 2011; 14:72-80.

31. Nishikawa K, Morita S, Matsui T, Kobayashi M, Takeuchi Y, Takahashi I, Sato S, Miyashita Y, Tsuburaya A, Sakamoto J, Kakeji Y, Baba H. A randomized phase-II trial comparing sequential and concurrent paclitaxel with oral or parenteral fluorinated pyrimidines for advanced or metastatic gastric cancer. Gastric Cancer. 2012; 15:363-369.

32. Popov I, Radosevic-Jelic L, Jezdic S, Milovic M, Borojevic N, Stojanovic S, Stankovic V, Josifovski T, Kezic I. Biweekly oxaliplatin, fluorouracil and leucovorin versus cisplatin, fluorouracil and leucovorin in patients with advanced gastric cancer. J BUON. 2008; 13:505-511.

33. Sawaki A, Yamaguchi K, Nabeya Y, Sakai Y, Osanai H, Denda T, Furue H, Kurihara M. 5-FU/1-LV (RPMI) versus $\mathrm{S}-1$ as first-line therapy in patients with advanced gastric cancer: A randomized phase III non-inferiority trial. (ISO5FU10 study group trial). European Journal of Cancer, Supplement. 2009; 7:364.

34. Seol YM, Song MK, Choi YJ, Kim GH, Shin HJ, Song GA, Chung JS, Cho GJ. Oral fluoropyrimidines (capecitabine or S-1) and cisplatin as first line treatment in elderly patients with advanced gastric cancer: a retrospective study. Jpn J Clin Oncol. 2009; 39:43-48.

35. Shen L, Jin M, Lu H, Li J, Chen Z, Shi Y, Song S, Qin S, Qin J, Ouyang X. Randomized 3-armed phase III study of S-1 monotherapy versus S-1/CDDP (SP) versus 5-FU/ CDDP (FP) in patients (pts)with advanced gastric cancer (AGC) (SC-101 study). Annals of Oncology. 2011; 22:ix24.
36. Shitara K, Sawaki A, Matsuo K, Kondo C, Takahari D, Ura T, Tajika M, Niwa Y, Muro K. A retrospective comparison of S-1 plus cisplatin and capecitabine plus cisplatin for patients with advanced or recurrent gastric cancer. Int J Clin Oncol. 2013; 18:539-546.

37. Sugimoto N, Fujitani K, Imamura H, Uedo N, Iijima S, Imano M, Shimokawa T, Kurokawa Y, Furukawa H, Goto M. Randomized phase II trial of S-1 plus irinotecan versus S-1 plus paclitaxel as first-line treatment for advanced gastric cancer (OGSG0402). Anticancer Res. 2014; 34:851-857.

38. Wang X, Wang ML, Zhou LY, Lu XY, Yang JF, Yu HG. Randomized phase II study comparing paclitaxel with S-1 vs. S-1 as first-line treatment in patients with advanced gastric cancer. Clin Transl Oncol. 2013; 15:836-842.

39. Xu RH, Sun GP, Lu HS, Peng LY, Xu JM, Zhong MZ, Zhang HL, Yu SY, Li W, Hu XH, Wang JJ, Cheng Y, Zhou JT, et al. A phase III study of S-1 plus cisplatin versus fluorouracil plus cisplatin in patients with advanced gastric or gastroesophageal junction adenocarcinoma. Journal of Clinical Oncology. 2013; 31.

40. Yamada Y, Higuchi K, Nishikawa K, Gotoh M, Fuse N, Sugimoto N, Nishina T, Amagai K, Chin K, Niwa Y, Tsuji A, Imamura H, Tsuda $\mathrm{M}$, et al. Phase III study comparing oxaliplatin plus S-1 with cisplatin plus S-1 in chemotherapy-naive patients with advanced gastric cancer. Ann Oncol. 2015; 26:141-148.

41. Yoshino S, Nishikawa K, Morita S, Takahashi T, Sakata K, Nagao J, Nemoto H, Murakami N, Matsuda T, Hasegawa H, Shimizu R, Yoshikawa T, Osanai H, et al. Randomised phase III study of S-1 alone versus S-1 plus lentinan for unresectable or recurrent gastric cancer (JFMC36-0701). European Journal of Cancer. 2016; 65:164-171.

42. Perego P, Robert J. Oxaliplatin in the era of personalized medicine: from mechanistic studies to clinical efficacy. Cancer Chemother Pharmacol. 2016; 77:5-18.

43. Di Francesco AM, Ruggiero A, Riccardi R. Cellular and molecular aspects of drugs of the future: oxaliplatin. Cell Mol Life Sci. 2002; 59:1914-1927.

44. Nishiyama M, Wada S. Docetaxel: its role in current and future treatments for advanced gastric cancer. Gastric Cancer. 2009; 12:132-141.

45. Tetzlaff ED, Cheng JD, Ajani JA. Review of docetaxel in the treatment of gastric cancer. Ther Clin Risk Manag. 2008; 4:999-1007.

46. Liu GF, Tang D, Li P, Wang S, Xu YX, Long AH, Zhou NL, Zhang LL, Chen J, Xiang XX. S-1-based combination therapy vs S-1 monotherapy in advanced gastric cancer: a meta-analysis. World J Gastroenterol. 2014; 20:310-318.

47. Zhu L, Liu J, Ma S. Fluoropyrimidine-Based Chemotherapy as First-Line Treatment for Advanced Gastric Cancer: a Bayesian Network Meta-Analysis. Pathol Oncol Res. 2016; 22:853-861. 